\title{
Projections Onto The Epigraph Set of The Filtered Variation Function Based Deconvolution Algorithm
}

\author{
Mohammad Tofighi and A. Enis Cetin ${ }^{\dagger}$ \\ Department of Electrical Engineering, Pennsylvania State University, State College, PA \\ ${ }^{\dagger}$ Department of Electrical and Electronics Engineering, Bilkent University, Ankara, Turkey \\ tofighi@psu.edu, ${ }^{\dagger}$ cetin@ @ilkent.edu.tr
}

\begin{abstract}
A new deconvolution algorithm based on orthogonal projections onto the hyperplanes and the epigraph set of a convex cost function is presented. In this algorithm, the convex sets corresponding to the cost function are defined by increasing the dimension of the minimization problem by one. The Filtered Variation (FV) function is used as the convex cost function in this algorithm. Since the FV cost function is a convex function in $\mathbb{R}^{N}$, then the corresponding epigraph set is also a convex set in the lifted set in $\mathbb{R}^{N+1}$. At each step of the iterative deconvolution algorithm, starting with an arbitrary initial estimate in $\mathbb{R}^{N+1}$, first the projections onto the hyperplanes are performed to obtain the first deconvolution estimate. Then an orthogonal projection is performed onto the epigraph set of the FV cost function, in order to regularize and denoise the deconvolution estimate, in a sequential manner. The algorithm converges to the deblurred image.
\end{abstract}

Keywords-Epigraph set of a convex cost function, deconvolution, projection onto convex sets, filtered variation

\section{INTRODUCTION}

A new deconvolution algorithm based on orthogonal Projections onto the Epigraph Set of a Convex cost function (PESC) is introduced [1, 2]. In Bregman's standard POCS approach [3], the algorithm converges to the intersection of convex constraint sets. In this article, it is shown that it is possible to use a convex cost function in a POCS based framework using the epigraph set and the new framework is used in deconvolution.

Bregman also developed iterative methods based on the so-called Bregman distance to solve convex optimization problems [3]. In Bregman's approach, it is necessary to perform a Bregman projection at each step of the algorithm, which may not be easy to compute the Bregman distance in general [4].

In standard POCS approach, the goal is simply to find a vector, which is in the intersection of convex constraint sets [5-14, 16, 17]. In each step of the iterative algorithm, an orthogonal projection is performed onto one of the convex sets. Bregman showed that successive orthogonal projections converge to a vector, which is in the intersection of all the convex sets. If the sets do not intersect iterates oscillate between members of the sets [20]. Since, there is no need to compute the Bregman distance in standard POCS, it found applications in many practical problems. In this article, orthogonal projections onto the epigraph set of a convex cost functions is used to solve convex optimization problems instead of the Bregman distance approach.

In the proposed deconvolution algorithm using PESC algorithm, first the projections onto deconvolution hyperplanes are performed, which results in the first deconvolution estimate. Then the deconvolution estimate is projected onto the epigraph set of FV function. This process will continue in an iterative manner till the deblurred image is obtained.

In PESC approach [1], in order to solve the signal reconstruction or restoration problem, the dimension is increased by one and sets corresponding to Filtered Variation (FV) cost function are defined. This approach is graphically illustrated in Fig.2. Since the FV cost function is a convex function in $\mathbb{R}^{N}$, then the corresponding epigraph set is also a convex set in $\mathbb{R}^{N+1}$. As a result, the convex minimization problem is reduced to finding the $\left[\mathbf{w}^{*}, f\left(\mathbf{w}^{*}\right)\right]$ vector of the epigraph set corresponding to the cost function as shown in Fig. 1. As in standard POCS approach, the new iterative optimization method starts with an arbitrary initial estimate in $\mathbb{R}^{N+1}$ and an orthogonal projection is performed onto one of the constraint sets. The resulting vector is then projected onto the epigraph set. This process is continued in a sequential manner at each step of the optimization problem. This method provides globally optimal solutions for convex cost functions such as total-variation [1, 21], filtered variation [22], $\ell_{1}$-norm [2], and entropic function [23].

The article is organized as follows. In Section II, the epigraph set of filtered variation function is defined and the convex minimization method based on the PESC approach is introduced. In Section III, the new deconvolution method is presented. The new approach does not require any regularization parameter as in other TV based methods [5, 11, 21]. In Section IV, the simulation results and some deconvolution examples are presented.

\section{EPIGRAPH SET OF Filtered VARIATION Function}

Let the original image be $\mathbf{v}$, and it is corrupted version by white Gaussian noise be $\mathbf{v}_{0}$. Suppose that the observation model is the additive noise model:

$$
\mathbf{v}_{0}=\mathbf{v}+\boldsymbol{\eta}
$$

where $\boldsymbol{\eta}$ is the additive Gaussian noise with variance $\sigma_{\eta}^{2}$.

It can be shown that the FV function $f(\mathbf{w}): \mathbb{R}^{N} \rightarrow \mathbb{R}$ is a convex cost function [22]. Different types of FV constraints are introduced in [22]. In this paper, for an image defined as $\mathbf{w}=\{w[i, j]: 1 \leq i, j \leq M\} \in \mathbb{R}^{M \times M}=\mathbb{R}^{N}$, we use the following definition for FV function:

$$
f(\mathbf{w})=\sum_{i, j}\left|\sum_{k, l} h[k, l] w[i-k, j-l]\right|,
$$


where $\mathbf{h}=\{h[k, l]:-r \leq k, l \leq r\} \in \mathbb{R}^{(2 r+1) \times(2 r+1)}$ is the high-pass filter with $r \ll M$. We define the epigraph set of the $\mathrm{FV}$ in $\mathbb{R}^{N+1}$ as follows:

$$
\mathrm{C}_{\mathrm{FV}}=\left\{\underline{\mathbf{w}}=\left[\begin{array}{ll}
\mathbf{w}^{T} & y
\end{array}\right]^{T}: y \geq f(\mathbf{w})\right\},
$$

where $f(\mathbf{w})$ is the $\mathrm{FV}$ function. $\mathrm{C}_{\mathrm{FV}}$ is the set of $N+1$ dimensional vectors, whose $(N+1)^{s t}$ component $y$ is greater than $f(\mathbf{w})$. We use bold face letters for $N$ dimensional vectors and underlined bold face letters for $N+1$ dimensional vectors, respectively. A graphical description of the epigraph concept is illustrated in Fig. 1.

The first step of our denoising algorithm consists of making an orthogonal projection onto $C_{\mathrm{FV}}$. Let $\underline{\mathbf{v}}_{0}=\left[\begin{array}{ll}\mathbf{v}_{0}^{T} & 0\end{array}\right]^{T}$ be an arbitrary vector in $\mathbb{R}^{N+1}$. The projection $\underline{\mathbf{w}}^{*}$ is determined by minimizing the distance between $\underline{\mathbf{v}}_{0}$ and $C_{\mathrm{FV}}$, i.e.,

$$
\underline{\mathbf{w}}^{*}=\arg \min _{\underline{\mathbf{w}}_{i} \in \mathrm{C}_{\mathrm{FV}}}\left\|\underline{\mathbf{v}}_{0}-\underline{\mathbf{w}}_{i}\right\|^{2} .
$$

In this approach, we project the vector version of $\mathbf{v}_{0}$ in (1) onto the $C_{\mathrm{FV}}$. This means that we select the nearest vector $\mathbf{w}^{\star}$ on the set $C_{\mathrm{FV}}$ to $\mathbf{v}_{0}$ as illustrated in Fig. 1. Equation (4) is equivalent to:

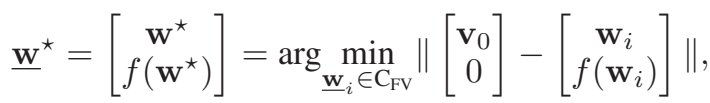

where $\underline{\mathbf{w}}^{\star}=\left[\mathbf{w}^{* T}, f\left(\mathbf{w}^{*}\right)\right]^{T}$ is the nearest vector to $\left[\mathbf{v}_{0}, 0\right]^{T}$ on the epigraph set. The projection $\underline{\mathbf{w}}^{\star}$ must be on the boundary of the epigraph set. Therefore, the projection must be of the form $\left[\mathbf{w}^{* T}, f\left(\mathbf{w}^{*}\right)\right]^{T}$. Eq. (5) becomes:

$$
\underline{\mathbf{w}}^{\star}=\left[\begin{array}{c}
\mathbf{w}^{*} \\
f\left(\mathbf{w}^{*}\right)
\end{array}\right]=\arg \min _{\underline{\mathbf{w}}_{i} \in \mathrm{C}_{\mathrm{FV}}}\left(\left\|\mathbf{v}_{0}-\mathbf{w}_{i}\right\|_{2}^{2}+f\left(\mathbf{w}_{i}\right)^{2}\right) .
$$

Solution of (6) using projections onto boundary and tangential hyperplanes are described in [1, 24], and we briefly explain those concepts in this section.

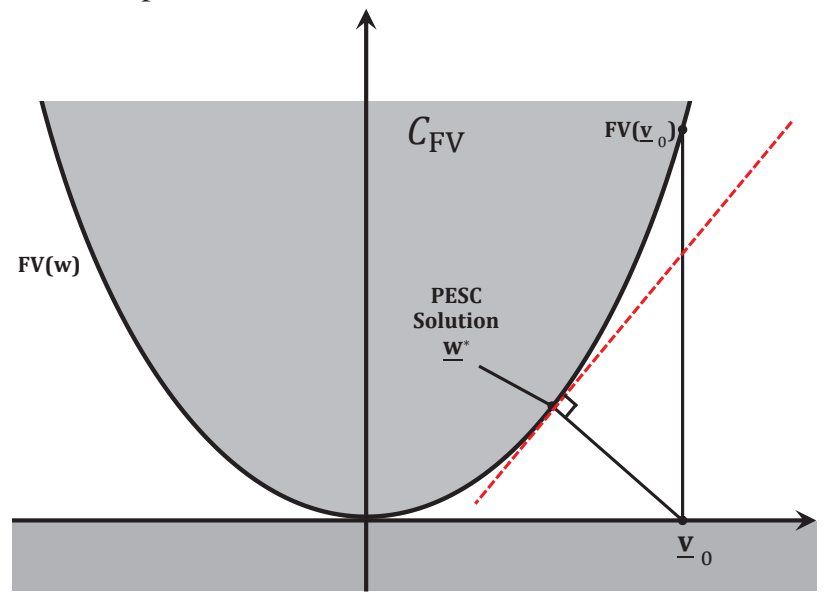

Fig. 1: Graphical representation of the minimization operation in (5), and (6). The corrupted observation vector $\mathbf{v}$ is projected onto the set $C_{\mathrm{FV}}$.

In the proposed method, the regularization term is the square of the FV function as shown in (6). The first term in (6) consists of components $\left|\mathrm{v}_{i}-\mathrm{w}_{i}\right|$ which are comparable to $\left|\mathrm{w}_{i}-\mathrm{w}_{i-1}\right|$ forming the FV function. The $\ell_{2}$-norm dominates the FV function in ordinary LASSO cost function. However, in (6) the square of $f(\mathbf{w})$ increases the effect of the regularization term. It also leads to an efficient computational solution in [1]. The PESC algorithm used in the proposed deconvolution algorithm is described in detail in next section.

Finding the right regularization parameter is a major problem in LASSO. Unlike LASSO approach [25], where the selection of the $\lambda$ parameters is determined in an ad-hoc manner or inspection, in the PESC based denoising algorithm, it is experimentally observed that $\lambda=1$ works well [24]. We tried various $\lambda$ values between 0.2 and 2 and $\lambda=1$ produced the best results. The PESC software is available in [24].

\section{DeCONVOLUtion Using PESC}

In this section, we present a new deconvolution method, based on the epigraph set of the Filtered variation function. Let the original signal or image be $\mathbf{w}_{\text {orig }}$ and its blurred and noisy version be $\mathbf{z}$ :

$$
\mathbf{z}=\mathbf{w}_{\text {orig }} * \mathbf{h}+\boldsymbol{\eta},
$$

where $\mathbf{h}$ is the point spread function (PSF) and $\boldsymbol{\eta}$ is the additive white Gaussian noise. In this approach, as in (4), we solve the following problem:

$$
\underline{\mathbf{w}}^{\star}=\underset{\underline{\mathbf{w}} \in \mathrm{C}_{\mathrm{f}}}{\arg \min }\left\|\underline{\mathbf{v}}_{0}-\underline{\mathbf{w}}\right\|^{2},
$$

where $\underline{\mathbf{v}}_{0}=\left[\begin{array}{ll}\mathbf{v}_{0}^{T} & 0\end{array}\right]^{T}$, and $\mathrm{C}_{\mathrm{FV}}$ is the epigraph set of $\mathrm{FV}$ in $\mathbb{R}^{N+1}$. To estimate this problem, we use PESC framework using the following sets:

$$
\mathrm{C}_{i}=\left\{\mathbf{w} \in \mathbb{R}^{N} \mid z_{i}=(\mathbf{w} * \mathbf{h})[i]\right\} \quad i=1,2, \ldots, L,
$$

where $L$ is the total number of pixels, $z_{i}$ is the $i^{t h}$ observation, and $\mathrm{C}_{i}$ is the set of the observation hyperplanes, and the epigraph set:

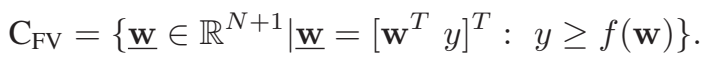

Notice that the sets $\mathrm{C}_{i}$ are in $\mathbb{R}^{N}$ and $\mathrm{C}_{\mathrm{FV}}$ is in $\mathbb{R}^{N+1}$. However, it is straightforward to extend $\mathrm{C}_{i}$ 's to $\mathbb{R}^{N+1}$ and they are still closed and convex sets in $\mathbb{R}^{N+1}$. Let us describe the projection operation onto the set $\mathrm{C}_{\mathrm{FV}}=\{\mathrm{FV}(\mathbf{w}) \leq y\}$. This means that we select the nearest vector $\underline{\mathbf{w}}^{\star}$ on the set $\mathrm{C}_{\mathrm{FV}}$ to $\mathbf{v}_{0}$. This is graphically illustrated in Fig. 2. During this orthogonal projection operations, we do not require any parameter adjustment as in [21]. The POCS algorithm consists of cyclical projections onto the sets $\mathrm{C}_{i}$ and $\mathrm{C}_{\mathrm{FV}}$.

Projection onto the sets are very easy to compute because they are hyperplanes. The projection equation is as follows:

$$
\mathbf{v}_{r+1}=\mathbf{v}_{r}+\frac{z_{i}-\left(\mathbf{v}_{r} * \mathbf{h}\right)[i]}{\|\mathbf{h}\|^{2}} \mathbf{h}^{T},
$$

where $\mathbf{v}_{r}$ is the estimated deblurred image in the $r^{t h}$ iterate, and $\mathbf{v}_{r+1}$ is the projection vector onto the hyperplane $\mathrm{C}_{i}$, then $\mathbf{v}_{r+1}$ is projected onto $\mathrm{C}_{\mathrm{FV}}$. The pseudo-code of the algorithm is described in Algorithm 1. The sets $\mathrm{C}_{i}$ and $\mathrm{C}_{\mathrm{FV}}$ may or may not intersect in $\mathbb{R}^{N+1}$. If they intersect, iterates converge to a solution in the intersection set. It is also possible to use hyperslabs $\mathrm{C}_{i, h}=\left\{\mathbf{w} \mid z_{i}-\epsilon_{i} \leq(\mathbf{w} * \mathbf{h})[i] \leq z_{i}+\epsilon_{i}\right\}$ instead of hyperplanes $\mathrm{C}_{i}$ in this algorithm. In this case, it is more likely 
that the closed and convex sets of the proposed framework intersect.

Implementation: The sub-gradient projections of $\mathbf{v}_{r}$ are performed as in Eq. 11. Then after a loop of these projections are terminated, and a deconvolution estimate is obtained, the PESC algorithm will be applied to the output $\mathbf{v}_{r+1}$. The projection operation described in Eq. (8) can not be obtained in one step when the cost function is FV. The solution is determined by performing successive orthogonal projections onto supporting hyperplanes of the epigraph set $\mathrm{C}_{\mathrm{FV}}$. In the first step, $f\left(\underline{\mathbf{v}}_{0}\right)$ and the surface normal at $\underline{\mathbf{v}}_{1}=\left[\mathbf{v}_{0}^{T} f\left(\mathbf{v}_{0}\right)\right]$ in $\mathbb{R}^{N+1}$ are calculated. In this way, the equation of the supporting hyperplane at $\underline{\mathbf{v}}_{1}$ is obtained. The vector $\mathbf{v}_{0}=\left[\begin{array}{ll}\mathbf{v}_{0}^{T} & 0\end{array}\right]$ is projected onto this hyperplane and $\underline{\mathbf{w}}_{0}$ is obtained as the first estimate as shown in Fig. 2. In the second step, $\underline{\mathbf{w}}_{0}$ is projected onto the level set, $\mathbf{C}_{s}=\left\{\underline{\mathbf{w}}=\left[\mathbf{w}^{T} y\right]^{T}, y \leq 0: y \geq f(\mathbf{w})\right\}$, by simply making its last component zero. The FV of this vector, the surface normal, and the supporting hyperplane are calculated as in the previous step. Next, $\underline{\mathbf{v}}_{2}$ is projected onto the new supporting hyperplane, and $\underline{\mathbf{w}}_{1}$ is obtained. In general, iterations continue until $\left\|\underline{\mathbf{w}}_{i}-\underline{\mathbf{w}}_{i-1}\right\| \leq \epsilon$, where $\epsilon$ is a prescribed number, or iterations can be stopped after a certain number of iterations.

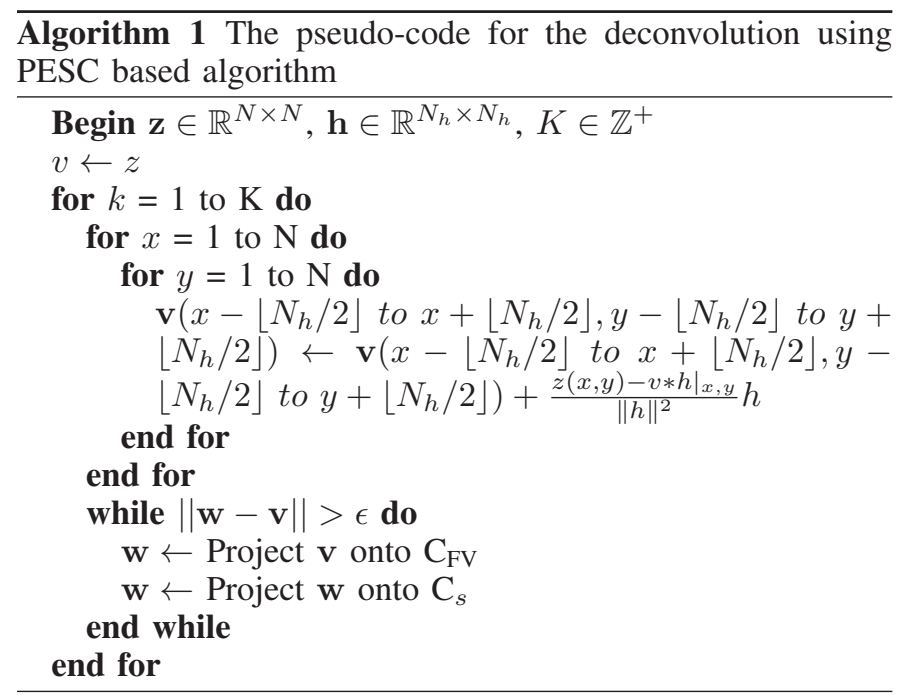

We calculate the distance between $\underline{\mathbf{v}}_{0}$ and the projection vector $\underline{\mathbf{w}}_{i}$ at each step of the iterative algorithm. The distance $\left\|\underline{\mathbf{v}}_{0}-\underline{\mathbf{w}}_{i}\right\|^{2}$ does not always decrease for high $i$ values. This happens around the optimal denoising solution $\underline{\mathbf{w}}^{\star}$. Once we detect an increase in $\left\|\underline{\mathbf{v}}_{0}-\underline{\mathbf{w}}_{i}\right\|^{2}$, we perform a refinement step to obtain the final solution of the denoising problem. In refinement step, the supporting hyperplane at $\underline{\mathbf{v}}_{2 i-1}=$ $\frac{\underline{\mathbf{v}}_{2 i-5}+\underline{\mathbf{v}}_{2 i-3}}{2}$ is used in the next iteration. For instance, in Fig. 2 , the supporting hyperplane at $\mathbf{v}_{5}$ is used in the next step as refinement step. A typical convergence graph is shown in Fig. 3 for a sample image. The proposed deconvolution method is described in Algorithm 1.

\section{Simulation Results}

In order to evaluate the proposed deconvolution algorithm, the simulation results for PESC and FTL [26] algorithms are presented for some image processing standard images and microscopic cancer cell images. The original image is blurred

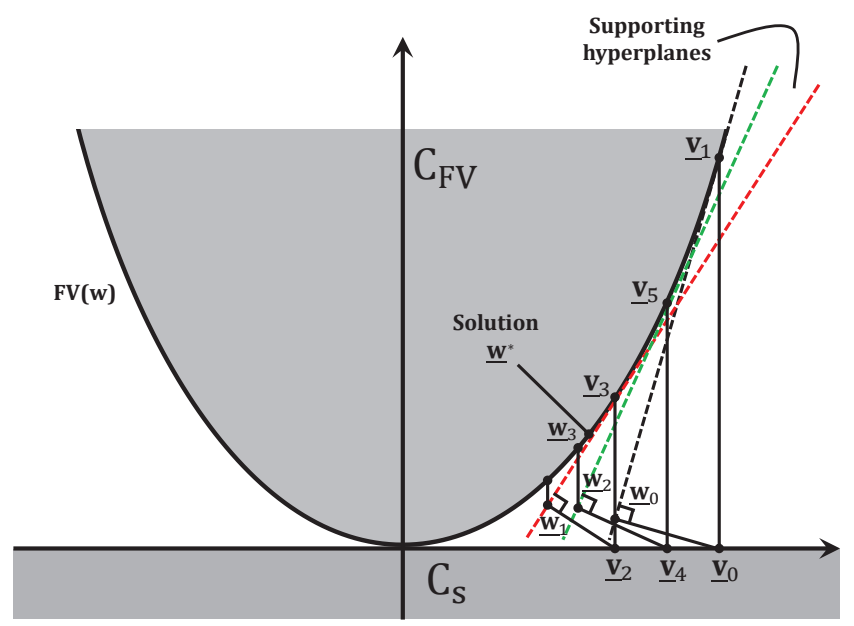

Fig. 2: Graphical representation of the minimization of Eq. (8), using projections onto the supporting hyperplanes of $\mathrm{C}_{\mathrm{FV}}$. In this problem the sets $C_{s}$ and $C_{F V}$ intersect because $f(\mathbf{w})=0$ for $\mathbf{w}=[0,0, \ldots, 0]^{T}$ or for a constant vector.

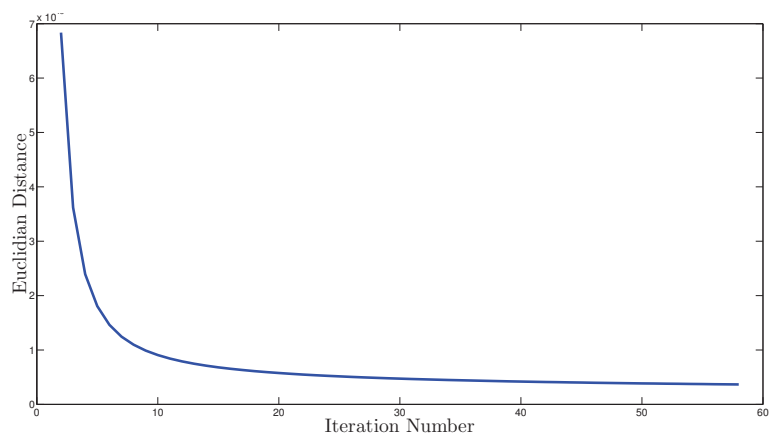

Fig. 3: Euclidian distance from $\mathbf{v}_{0}$ to the epigraph of $\mathrm{FV}$ at each iteration $\left(\left\|\underline{\mathbf{v}}_{0}-\underline{\mathbf{w}}_{i}\right\|\right)$ with noise standard deviation of $\sigma=30$.

with $9 \times 9$ uniform blurring matrix. Then it is corrupted with additive white Gaussian noise with variance $\sigma_{\eta}^{2}$. The noise variance, $\sigma_{\eta}^{2}$, is chosen such that the Blurred Signal to Noise Ratio (BSNR) reaches a target value. The BSNR value is calculated as follows:

$$
\mathrm{BSNR}=10 \times \log _{10}\left(\frac{\|\tilde{\mathbf{z}}-E[\tilde{\mathbf{z}}]\|^{2}}{N \sigma_{\eta}^{2}}\right),
$$

where $\tilde{\mathbf{z}}$ is the blurred image without noise: $\tilde{\mathbf{z}}=\mathbf{w}_{\text {orig }} * \mathbf{h}, N$ is the total number of pixels, $\sigma_{\eta}$ is the standard deviation of the additive noise, and $E[\cdot]$ indicates the mean value. In addition to the visual results, the deblurring algorithms are compared in terms of Improved Signal to Noise Ratio (ISNR) and Signal to Noise Ratio (SNR). The SNR and ISNR values are calculated as follows:

$$
\begin{gathered}
\text { SNR }=10 \times \log _{10}\left(\frac{\left\|\mathbf{w}_{\text {orig }}\right\|^{2}}{\left\|\mathbf{z}-\mathbf{w}_{\text {orig }}\right\|^{2}}\right), \\
\text { ISNR }=10 \times \log _{10}\left(\frac{\left\|\mathbf{z}-\mathbf{w}_{\text {orig }}\right\|^{2}}{\left\|\mathbf{w}_{\text {rec }}-\mathbf{w}_{\text {orig }}\right\|^{2}}\right),
\end{gathered}
$$

which $\mathbf{w}_{\text {rec }}$ is the reconstructed and deblurred image. The ISNR vs. iteration number for the MRI image is presented in Fig. 5. 


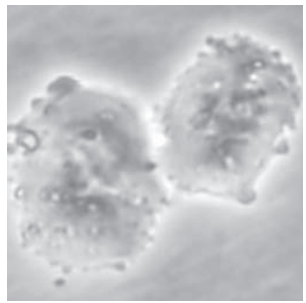

(a) Original

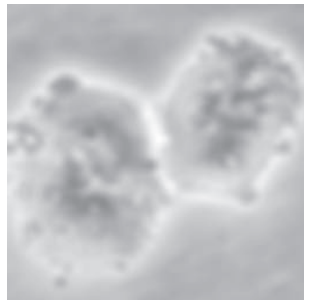

(b) Blurred

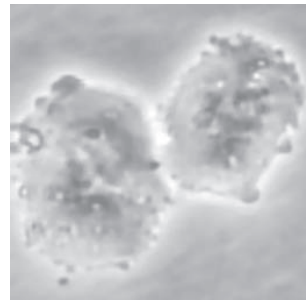

(c) PESC

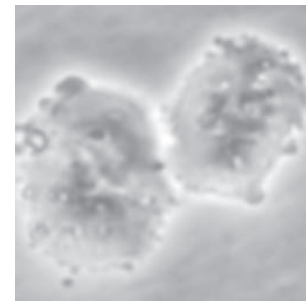

(d) FTL

Fig. 4: Cancer cell image (a) Original, (b) Blurred (BSNR $=50$ ), (c) Deblurred by PESC (SNR $=40.58 \mathrm{~dB}$ ), (d) Deblurred by FTL $(\mathrm{SNR}=39.35 \mathrm{~dB})$.

TABLE I: ISNR and SNR results for PESC based deconvolution algorithm.

\begin{tabular}{|c|c|c|c|c|c|c|c|c|c|c|c|c|c|c|}
\hline BSNR & \multicolumn{2}{|c|}{ Cameraman } & \multicolumn{2}{|c|}{ Lena } & \multicolumn{2}{|c|}{ Peppers } & \multicolumn{2}{|c|}{ Pirate } & \multicolumn{2}{|c|}{ Mandrill } & \multicolumn{2}{|c|}{ MRI } & \multicolumn{2}{|c|}{ Cancer cell } \\
\hline 30 & 5.59 & 20.83 & 4.48 & 24.94 & 5.35 & 26.13 & .57 & 22.77 & $\overline{4.56}$ & 20.77 & .64 & 13.71 & 6.26 & 35.94 \\
\hline 35 & 7.01 & & 5.7 & & 5.88 & & .5 & & 0.61 & & 5.76 & & 7.76 & 7.69 \\
\hline 40 & 8.49 & 23.77 & 6.95 & 27.46 & 7.45 & 28.3 & 6.75 & 24.99 & 6.4 & 22.6 & 7.07 & 16.28 & 9.05 & 38.79 \\
\hline 45 & 9.75 & 25.04 & 8.03 & 28.55 & 8.52 & 29.39 & 7.87 & 26.12 & 6.72 & 22.95 & 8.40 & 22.95 & 9.76 & 39.51 \\
\hline 50 & 10.76 & 26.10 & 8.49 & 29.00 & 9.50 & 30.37 & 8.41 & 26.66 & 6.84 & 23.07 & 9.31 & 18.53 & 10.83 & 40.58 \\
\hline
\end{tabular}

TABLE II: ISNR and SNR results for FTL based deconvolution algorithm.

\begin{tabular}{|c|c|c|c|c|c|c|c|c|c|c|c|c|c|c|}
\hline BSNR & \multicolumn{2}{|c|}{ Cameraman } & \multicolumn{2}{|c|}{ Lena } & \multicolumn{2}{|c|}{ Peppers } & \multicolumn{2}{|c|}{ Pirate } & \multicolumn{2}{|c|}{ Mandrill } & \multicolumn{2}{|c|}{ MRI } & \multicolumn{2}{|c|}{ Cancer cell } \\
\hline 30 & $\begin{array}{l}-0.4 \\
\end{array}$ & 14.79 & $\overline{-0.74}$ & 19.7 & -3.26 & 17.2 & 0.71 & 18.74 & 4.32 & 20.52 & 4.08 & 13.02 & 4.66 & 34.34 \\
\hline 35 & 6.16 & 21.35 & 5.46 & 25.97 & 5.66 & 26.11 & 5.61 & 23.68 & 5.45 & 21.65 & 5.03 & 14.36 & 7.65 & 37.58 \\
\hline 40 & 7.54 & 22.73 & 6.60 & 27.13 & 8.00 & 28.45 & 6.44 & 24.50 & 5.74 & 21.94 & 5.45 & 14.73 & 8.98 & 38.72 \\
\hline 45 & 7.89 & 23.08 & 6.93 & 27.46 & 8.14 & 29.02 & 6.67 & 24.75 & 5.89 & 22.08 & 5.56 & 14.86 & 9.44 & 39.19 \\
\hline 50 & 8.04 & 23.23 & 7.07 & 27.59 & 8.74 & 29.20 & 6.77 & 24.84 & 5.98 & 22.18 & 5.61 & 14.92 & 9.59 & 39.35 \\
\hline
\end{tabular}

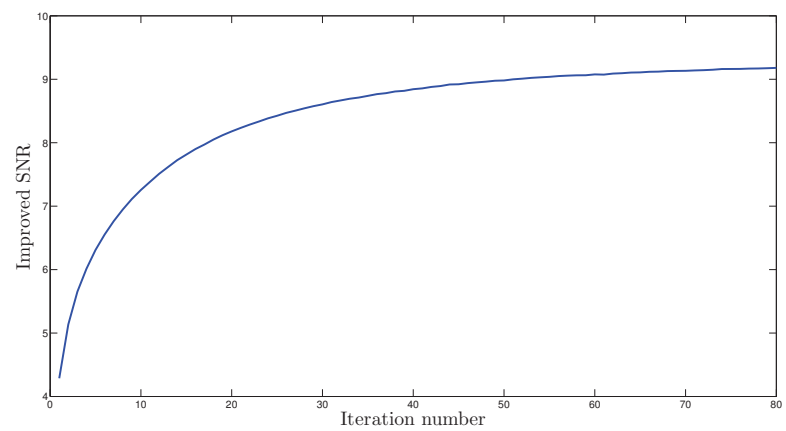

Fig. 5: ISNR vs. iteration number for MRI image (BSNR = $50)$.

Table I and II represent the ISNR and SNR values for five BSNR levels for PESC algorithm and FTL algorithm proposed by Vonesch et al. [26] for seven different images. Table III represents SNR and ISNR values for five different microscopic cancer cell images for PESC and FTL algorithms for BSNR = 45. According to these tables, in almost all cases PESC based deconvolution algorithm performs better than FTL [26] in the sense of ISNR and SNR, considering that the simulation time is similar for both algorithms.

In Fig. 4 the results for cancer cell image is presented. The original image is blurred with $9 \times 9$ uniform blurring matrix and is corrupted with additive white Gaussian noise with such a variance to obtain BSNR $=50$ value. The blurred image, and the deblurred images for both algorithms are presented in Fig. 4. According to these images, PESC algorithm performs better than FTL not only in sense of SNR, but also the results for PESC are visually better than FTL.

TABLE III: ISNR and SNR results for PESC and FTL based deconvolution algorithms for BSNR $=45$.

\begin{tabular}{|c|c|c|c|c|}
\hline Image & \multicolumn{2}{|c|}{ PESC } & \multicolumn{2}{c|}{ FTL } \\
\hline \hline Cancer cell-1 & $\mathbf{9 . 7 1}$ & $\mathbf{4 2 . 4 0}$ & 8.23 & 40.91 \\
\hline Cancer cell-2 & $\mathbf{1 0 . 4 7}$ & $\mathbf{4 1 . 8 7}$ & 8.79 & 40.16 \\
\hline Cancer cell-3 & $\mathbf{1 0 . 5 5}$ & $\mathbf{4 0 . 8 6}$ & 8.93 & 39.22 \\
\hline Cancer cell-4 & $\mathbf{9 . 0 2}$ & $\mathbf{4 2 . 0 9}$ & 7.63 & 40.73 \\
\hline Cancer cell-5 & $\mathbf{9 . 2 3}$ & $\mathbf{4 2 . 8 1}$ & 7.86 & 41.43 \\
\hline
\end{tabular}

\section{CONCLUSion}

A new deconvolution method based on the epigraph of the FV function is developed. Epigraph sets of other convex cost functions can be also used in the new deconvolution approach. The reconstructed signal is obtained by making an orthogonal projection onto the epigraph set from the corrupted signal in $\mathbb{R}^{N+1}$. The new algorithm does not need the optimization of the regularization parameter as in standard TV methods. Experimental results indicate that better SNR results are obtained compared to standard deconvolution in a large range of images. 


\section{REFERENCES}

[1] Mohammad Tofighi, Kivanc Kose, and A. Enis Cetin, "Denoising images corrupted by impulsive noise using projections onto the epigraph set of the total variation function (pes-tv)," Signal, Image and Video Processing, vol. 9, no. 1, pp. 41-48, 2015.

[2] A. Cetin and M. Tofighi, "Projection-based wavelet denoising [lecture notes]," Signal Processing Magazine, IEEE, vol. 32, no. 5, pp. 120-124, Sept 2015.

[3] L.M. Bregman, "Finding the common point of convex sets by the method of successive projection.(russian)," $\{$ USSR $\}$ Doklady Akademii Nauk SSSR, vol. 7, no. 3, pp. $200-217,1965$.

[4] W. Yin, S. Osher, D. Goldfarb, and J. Darbon, "Bregman iterative algorithms for $\ell_{1}$-minimization with applications to compressed sensing," SIAM Journal on Imaging Sciences, vol. 1, no. 1, pp. 143-168, 2008.

[5] S. Ono, M. Yamagishi, and I. Yamada, "A sparse system identification by using adaptively-weighted total variation via a primal-dual splitting approach," in IEEE ICASSP, 2013, pp. 6029-6033.

[6] Y. Censor, W. Chen, P. L. Combettes, R. Davidi, and G. T. Herman, "On the Effectiveness of Projection Methods for Convex Feasibility Problems with Linear Inequality Constraints," Computational Optimization and Applications, vol. 51, pp. 1065-1088, 2012.

[7] K. Slavakis, S. Theodoridis, and I. Yamada, "Online Kernel-Based Classification Using Adaptive Projection Algorithms," IEEE Transactions on Signal Processing, vol. 56, pp. 2781-2796, 2008.

[8] Y. Censor and A. Lent, "An Iterative Row-Action Method for Interval Convex Programming," Journal of Optimization Theory and Applications, vol. 34, pp. 321353, 1981.

[9] K. S Theodoridis and I. Yamada, "Adaptive learning in a world of projections," IEEE Signal Processing Magazine, vol. 28, no. 1, pp. 97-123, 2011.

[10] H. J. Trussell and M. R. Civanlar, "The Landweber Iteration and Projection Onto Convex Set," IEEE Transactions on Acoustics, Speech and Signal Processing, vol. 33, no. 6, pp. 1632-1634, 1985.

[11] P. L. Combettes and J.-Ch. Pesquet, "Image restoration subject to a total variation constraint," IEEE Transactions on Image Processing, vol. 13, pp. 1213-1222, 2004.

[12] P. L. Combettes, "The foundations of set theoretic estimation," Proceedings of the IEEE, vol. 81, pp. 182 $-208,1993$.

[13] H. S. Mousavi, V. Monga, and T. D. Tran, "Iterative convex refinement for sparse recovery," IEEE Signal Processing Letters, vol. 22, no. 11, pp. 1903-1907, Nov 2015.

[14] I. Yamada, M. Yukawa, and M. Yamagishi, "Minimizing the moreau envelope of nonsmooth convex functions over the fixed point set of certain quasi-nonexpansive mappings," Springer NY, pp. 345-390, 2011.

[15] I. Sezan and H. Stark, "Image restoration by the method of convex projections: Part 2-applications and numerical results," IEEE Transactions on Medical Imaging, vol. 1, pp. 95-101, 1982.

[16] Y. Censor and S. A. Zenios, "Proximal minimization algorithm withd-functions," Journal of Optimization Theory and Applications, vol. 73, pp. 451-464, 1992.

[17] A. Lent and H. Tuy, "An Iterative Method for the Extrapolation of Band-Limited Functions," Journal of Optimization Theory and Applications, vol. 83, pp. 554565,1981

[18] M. Rossi, A. M. Haimovich, and Y. C. Eldar, "Conditions for Target Recovery in Spatial Compressive Sensing for
MIMO Radar," IEEE ICASSP, 2013.

[19] L.G. Gubin, B.T. Polyak, and E.V. Raik, "The Method of Projections for Finding the Common Point of Convex Sets," Computational Mathematics and Mathematical Physics, vol. 7, pp. 1 - 24, 1967.

[20] A. E. Çetin, O.N. Gerek, and Y. Yardimci, "Equiripple FIR Filter Design by the FFT Algorithm," IEEE Signal Processing Magazine, vol. 14, no. 2, pp. 60-64, 1997.

[21] A. Chambolle, "An algorithm for total variation minimization and applications," Journal of Mathematical Imaging and Vision, vol. 20, no. 1-2, pp. 89-97, Jan. 2004.

[22] K. Kose, V. Cevher, and A.E. Cetin, "Filtered variation method for denoising and sparse signal processing," IEEE ICASSP, pp. 3329-3332, 2012.

[23] K. Kose, O. Gunay, and A. E. Cetin, "Compressive sensing using the modified entropy functional," Digital Signal Processing, 2013.

[24] "PES-TV software," http://signal.ee.bilkent.edu.tr/ PES-TV.html/.

[25] I. Johnstone B. Efron, T. Hastie and R. Tibshirani, "Least angle regression," Annals of Statistics, vol. 32, no. 2, pp. 407-499, 2004.

[26] C. Vonesch and M. Unser, "A fast thresholded landweber algorithm for wavelet-regularized multidimensional deconvolution," Image Processing, IEEE Transactions on, vol. 17, no. 4, pp. 539-549, April 2008. 